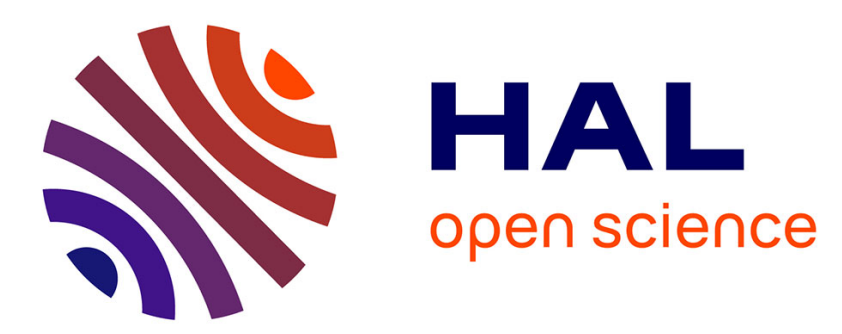

\title{
Elaboration d'une stratégie de management du talent: quelques conditions de réussite illustrées par une recherche-action
}

\author{
Mickaël Naulleau
}

\section{To cite this version:}

Mickaël Naulleau. Elaboration d'une stratégie de management du talent: quelques conditions de réussite illustrées par une recherche-action. Finance Contrôle Stratégie, 2015, 18 (3). hal-01221207

HAL Id: hal-01221207

https://hal-audencia.archives-ouvertes.fr/hal-01221207

Submitted on 27 Oct 2015

HAL is a multi-disciplinary open access archive for the deposit and dissemination of scientific research documents, whether they are published or not. The documents may come from teaching and research institutions in France or abroad, or from public or private research centers.
L'archive ouverte pluridisciplinaire HAL, est destinée au dépôt et à la diffusion de documents scientifiques de niveau recherche, publiés ou non, émanant des établissements d'enseignement et de recherche français ou étrangers, des laboratoires publics ou privés. 
18-3 (2015)

Varia

Mickael Naulleau

\title{
Elaboration d'une stratégie de management du talent : quelques conditions de réussite illustrées par une recherche-action
}

\begin{abstract}
Avertissement
Le contenu de ce site relève de la législation française sur la propriété intellectuelle et est la propriété exclusive de l'éditeur.

Les œuvres figurant sur ce site peuvent être consultées et reproduites sur un support papier ou numérique sous réserve qu'elles soient strictement réservées à un usage soit personnel, soit scientifique ou pédagogique excluant toute exploitation commerciale. La reproduction devra obligatoirement mentionner l'éditeur, le nom de la revue, l'auteur et la référence du document.

Toute autre reproduction est interdite sauf accord préalable de l'éditeur, en dehors des cas prévus par la législation en vigueur en France.
\end{abstract}

\section{revues.org}

Revues.org est un portail de revues en sciences humaines et sociales développé par le Cléo, Centre pour l'édition électronique ouverte (CNRS, EHESS, UP, UAPV).

\section{Référence électronique}

Mickael Naulleau, «Elaboration d'une stratégie de management du talent : quelques conditions de réussite illustrées par une recherche-action », Finance Contrôle Stratégie [En ligne], 18-3 | 2015, mis en ligne le 26 octobre 2015, consulté le 27 octobre 2015. URL : http://fcs.revues.org/1659

Éditeur : Association FCS

http://fcs.revues.org

http://www.revues.org

Document accessible en ligne sur :

http://fcs.revues.org/1659

Document généré automatiquement le 27 octobre 2015.

Tous droits réservés 


\section{Mickael Naulleau}

\section{Elaboration d'une stratégie de management du talent : quelques conditions de réussite illustrées par une recherche-action}

\section{Introduction}

Le Management du Talent (TM) connaît un large succès ces dernières années depuis la célèbre annonce du cabinet McKinsey sur la « guerre du talent» (Michaels et al. 2001). Soumises à la mondialisation d'une économie marquée par de profondes incertitudes, les entreprises européennes et internationales considèrent le TM comme l'enjeu prioritaire de gestion des ressources humaines qui leur permettra de surmonter les défis posés par ce nouvel environnement (Strack 2011). Cependant, à la fois parce qu'il reste à ce jour un champ d'étude fortement marqué par le contexte anglo-saxon et largement inspiré par des études de cas réalisés auprès d'entreprises multinationales du secteur privé, le TM reste aujourd'hui encore à l'âge de l'adolescence de sa maturité académique (Collings et al. 2011 ; Thunnissen et al. 2013 ; Vaiman et Collings 2013).

Trois thèmes de recherche principaux dominent la littérature sur le TM (Thunnissen et al. 2013). Le premier cherche à définir une notion de «talent » dont les travaux regrettent l'absence de définition claire et consistante (Al Ariss et al. 2014 ; Cappelli et Keller 2014 ; Lewis et Heckman 2006 ; Nijs et al. 2014 ; Vaiman et al. 2012) ainsi que la fragilité empirique de son cadre conceptuel (Collings et Mellahi 2009 ; Lewis et Heckman 2006). Le second porte sur les résultats attendus du TM en termes de motivation, d'engagement, de confiance ou encore de bien-être individuel, mais aussi de performance ou de compétitivité organisationnelle (Cappelli 2008 ; Collings et Mellahi 2009). Le dernier, enfin, centre son attention sur les processus du TM, à savoir l'ensemble des pratiques d'attraction, de développement et de rétention des talents développées par les organisations (Cappelli et Keller 2014 ; Stahl et al. 2012).

Ces travaux sur les processus du TM rejoignent le courant de recherche sur le «management stratégique du talent » (Collings et Mellahi 2009 ; Lewis et Heckman 2006) qui est issu des principes de la stratégie, de l'économie et des ressources humaines. Celui-ci propose une démarche de talentship fondée sur un modèle décisionnel permettant de structurer la stratégie du TM. Le talentship centre ainsi son attention sur le processus de mise en œuvre de cette stratégie du TM en laissant supposer à la fois un alignement exclusif du TM à la stratégie de l'entreprise et une capacité de toutes organisations à implémenter ce type de dispositif (Thunnissen et al. 2013).

Cet article interroge ces éléments à partir d'une perspective de recherche étonnamment absente dans la littérature qui est axée sur les antécédents du processus d'implémentation issu du modèle du talentship. Il centre plus précisément son attention sur le processus d'élaboration d'une stratégie de TM qui d'une part repose sur une notion théorique polysémique et qui, d'autre part, est soumis aux contingences organisationnelles propres au contexte dans lequel il prend place. Si cette approche nous paraît de nature à éclairer certaines pistes de recherche méconnues par la littérature sur le TM (Al Ariss et al. 2014), elle nous semble également pouvoir nous renseigner à la fois sur l'exclusivité supposée de l'alignement du TM à la stratégie de l'organisation, et sur les capacités organisationnelles (Joyce et Slocum 2012) nécessaires aux organisations pour s'engager dans une stratégie de TM.

De manière à mener une réflexion émergeante sur ce processus d'élaboration d'une stratégie de TM, nous avons adopté une démarche de recherche exploratoire. Celle-ci a été initiée par une demande d'aide à l'élaboration et à la mise en œuvre d'une stratégie du TM de la part du Directoire d'une Entreprise de Taille Intermédiaire (ETI) française (3 000 salariés). Cette 
demande nous a mené à réaliser une recherche-action (Coghlan 2011 ; Eden et Huxham 1996 ; Lewin 1946 ; Rapoport 1970 ; Susman et Evered 1978) fondée sur une relation collaborative ayant pour objectif à la fois d'apporter des solutions pratiques au problème de l'ETI et de générer des connaissances théoriques sur cet axe de recherche émergeant du TM. Notre objectif de recherche visait ainsi en particulier à questionner les présupposés du talentship lors du processus d'élaboration d'une stratégie de TM en répondant à la question de recherche suivante : quels sont les facteurs organisationnels de contingence au processus d'élaboration d'une stratégie de TM?

L'article positionne dans un premier temps notre problématique de recherche dans le champ des travaux sur le management stratégique du talent. Il précise ensuite la méthodologie de recherche-action employée dans le cas spécifique du groupe Régional. Il dresse alors les résultats à partir desquels est discutée une proposition de cadre d'analyse complémentaire au talentship permettant, lors du processus d'élaboration d'une stratégie de TM, d'évaluer les capacités organisationnelles requises au succès de son implémentation.

\section{Revue de littérature}

Le « talent » est une notion qui fait l'objet de nombreux débats dans la littérature. Il nous paraît utile de rendre compte de son inconsistance théorique pour souligner la fragilité d'une science de la décision du management stratégique du TM sur laquelle elle est construite. Nous discuterons alors quelques unes des limites du management stratégique du TM et du talentship pour positionner la problématique de recherche à laquelle souhaite répondre cet article.

\subsection{Une inconsistance théorique des notions liées au talent}

Deux approches se distinguent dans la littérature pour définir le talent (Gallardo-Gallardo et al. 2012 ; Stahl et al. 2012). La première fait référence à la personne (sujet talentueux, le Talent) et la seconde aux caractéristiques de la personne (objet, les talents). La première approche distingue elle-même deux types d'interprétation, l'une qualifiée d'inclusive et qui considère que tout le monde est talentueux, l'autre d'exclusive et qui estime que seule une élite de personnes est talentueuse. On observe cependant un consensus autour d'une définition exclusive où le talent d'un individu résulte d'une combinaison rare de compétences rares (Thévenet et Dejoux 2010) et d'un amalgame complexe et dynamique qui se compose d'aptitudes, de capacités cognitives, de connaissances, d'intelligence, de compétences, de potentialités (à grandir, à apprendre, à faire, etc.), de performance, de leadership, de créativité ou encore -sans que cette liste soit exhaustive- d'initiatives voire de traits de caractère (Michaels et al. 2001 ; Silzer et Church 2009 ; Tansley 2011).

Ces études soulignent également la dimension contextuelle d'une interprétation sur le talent (Gallardo-Gallardo et al. 2012 ; Nijs et al. 2014 ; Tansley 2011). Tansley (2011) montre par exemple que cette interprétation est davantage liée en Europe à une représentation du talent en tant que « douance » et somme de capacités innées manifestées dans un champ d'activité particulier (comme le sport, l'art, la recherche, le business, etc.) alors qu'elle est plutôt associée à une qualité pouvant être acquise et développée par la pratique dans les cultures non européennes. Ceci a ainsi ouvert la voie à des travaux questionnant le caractère inné ou acquis du talent (Gallardo-Gallardo et al. 2012 ; Meyers et al. 2013). De plus, Tansley (2011) a souligné la différence d'interprétation du talent selon le type d'organisation (centralisées, transnationales ou multinationales, ou bien encore de petites entreprises) ou encore le niveau organisationnel et managérial auquel on se situe.

La littérature fait ainsi l'aveu d'une impossibilité à donner une définition universelle du talent (Gallardo-Gallardo et al. 2012 ; Lewis et Heckman 2006 ; Tansley 2011 ; Thunnissen et al. 2013). Malgré tout, le positionnement philosophique à l'égard du talent (Meyers et van Woerkom 2014) est essentiel car il conditionne la nature et les modes de management implémentés pour le gérer (McDonnell 2011). Une perspective inclusive induit par exemple un mode de gestion applicable à l'ensemble des employés ; ce qui renforce le sentiment d'indifférenciation du TM avec les modes de GRH traditionnelles (Lewis et Heckman 2006). Une perspective exclusive nécessite au contraire un mode de gestion différencié qui peut 
utiliser les mêmes outils que la GRH mais se limiter à une population restreinte d'employés talentueux.

Cette indifférenciation entre GRH et TM fait ainsi l'objet de nombreux débats dans la communauté scientifique (Lewis et Heckman 2006). Certains (Chuai et al. 2008) considèrent que le TM n'est qu'un déguisement sophistiqué de pratiques RH traditionnelles (planification des effectifs, plan de succession, développement des salariés ou encore gestion de carrière) alors que d'autres pensent qu'il s'agit d'une perspective novatrice fondée sur un état d'esprit où le talent représente la clé de compétitivité organisationnelle (Cappelli 2008). La plupart des travaux s'accordent sur cette dernière approche de nature exclusive et définissent le TM comme «les activités et processus qui impliquent l'identification systématique des positions clés qui contribuent différemment à un avantage compétitif durable de l'organisation, au développement d'un 'pool' de talent de haut-potentiels et d'éléments hautement performants pour tenir ces positions (ou rôles), et le développement d'une architecture des ressources humaines différenciée pour faciliter le remplissage de ces positions avec des éléments compétents, et pour assurer leur implication continue à l'égard de l'organisation » (Collings et Mellahi 2009, p. 304).

Cette définition souligne la dimension «stratégique » de la pratique du TM dans le sens où celle-ci devrait débuter, selon les auteurs, par l'identification des positions clés ou des rôles critiques de l'organisation. Cette perspective conduit par conséquent à une distinction des rôles stratégiques et non-stratégiques dans l'organisation qui donne lieu à l'implémentation d'architectures de GRH différenciées (Al Ariss et al. 2014) : la GRH classique pour la gestion des emplois et compétences non-stratégiques, et le TM pour la gestion des postes et des talents stratégiques. Le TM repose alors sur l'ensemble des processus (attraction, sélection, développement et rétention) visant à anticiper les besoins en talents pour occuper ces emplois stratégiques (Cappelli et Keller 2014 ; Vaiman et al. 2012).

\subsection{Une science de la décision en matière de stratégie du TM}

Le TM se définit donc, dans cette perspective, comme une activité stratégique distincte de la GRH classique appelant à une attention soutenue aux quelques employés et positions qui ont un impact différentiel sur la stratégie de l'organisation. La question est donc de savoir, pour les décisionnaires, quels sont les rôles qui ont la contribution stratégique la plus importante, pour pouvoir ensuite définir les processus d'allocation de ressources optimaux. C'est à cette problématique d'opérationnalisation du TM en lien avec la stratégie que se sont attachés à répondre plusieurs travaux sur le management stratégique du talent.

Le management stratégique du talent est organisée autour d'une architecture RH spécifique (Collings et Mellahi 2009) articulant « une série intégrée de processus, de programmes et de normes culturelles dans une organisation (...) pour attirer, développer, déployer et retenir le talent en vue d'atteindre les objectifs stratégiques et répondre aux futurs besoins du business » (Silzer et Church 2009, p. 380). Cette approche exclusive du talent se centre sur les employés à haute-performance ou à haut-potentiel qui opèrent ou devront opérer dans des rôles clés ou pivots de l'organisation (Collings et Mellahi 2009). L'architecture du système de TM doit ainsi permettre à la fois d'identifier et d'alimenter les positions clés/pivots de l'organisation en talents clés/pivots au regard de la stratégie de l'organisation (Collings et Mellahi 2009 ; Lewis et Heckman 2006).

15 Sur la base des principes de la stratégie, de l'économie ou encore des ressources humaines, Boudreau et Ramstad (2004) ont développé une science de la décision, nommée talentship, qui se donne pour objectif «d'augmenter le succès de l'organisation en améliorant les décisions qui dépendent ou impactent les ressources en talent » (Boudreau et Ramstad 2007, p. 25). L'architecture du TM, qui doit permettre d'anticiper les besoins en talents (au regard de la stratégie de l'entreprise) et autour de laquelle doivent s'organiser les pratiques pour y parvenir (Cappelli 2008), s'élabore ainsi autour d'une série de décisions à prendre à trois niveaux d'analyse indépendants (Lewis et Heckman 2006) : 
- L'impact stratégique d'un vivier de talents, c'est-à-dire la manière dont un vivier de talents permet à l'organisation d'accomplir ses principaux buts stratégiques, qui conduit à une segmentation organisationnelle en différents viviers de talents pivots.

- L'efficacité qui renvoie au degré par lequel les pratiques (formation, coaching, etc.) affectent les comportements de ceux qui sont dans le vivier de talent ciblé.

- L'efficience qui est la mesure de la quantité d'activités (nombre de personnes formées, coût par recrutement, etc.) générée par l'investissement du TM.

Figure 1. Les 5 composantes du talentship (Lewis et Heckman 2006)

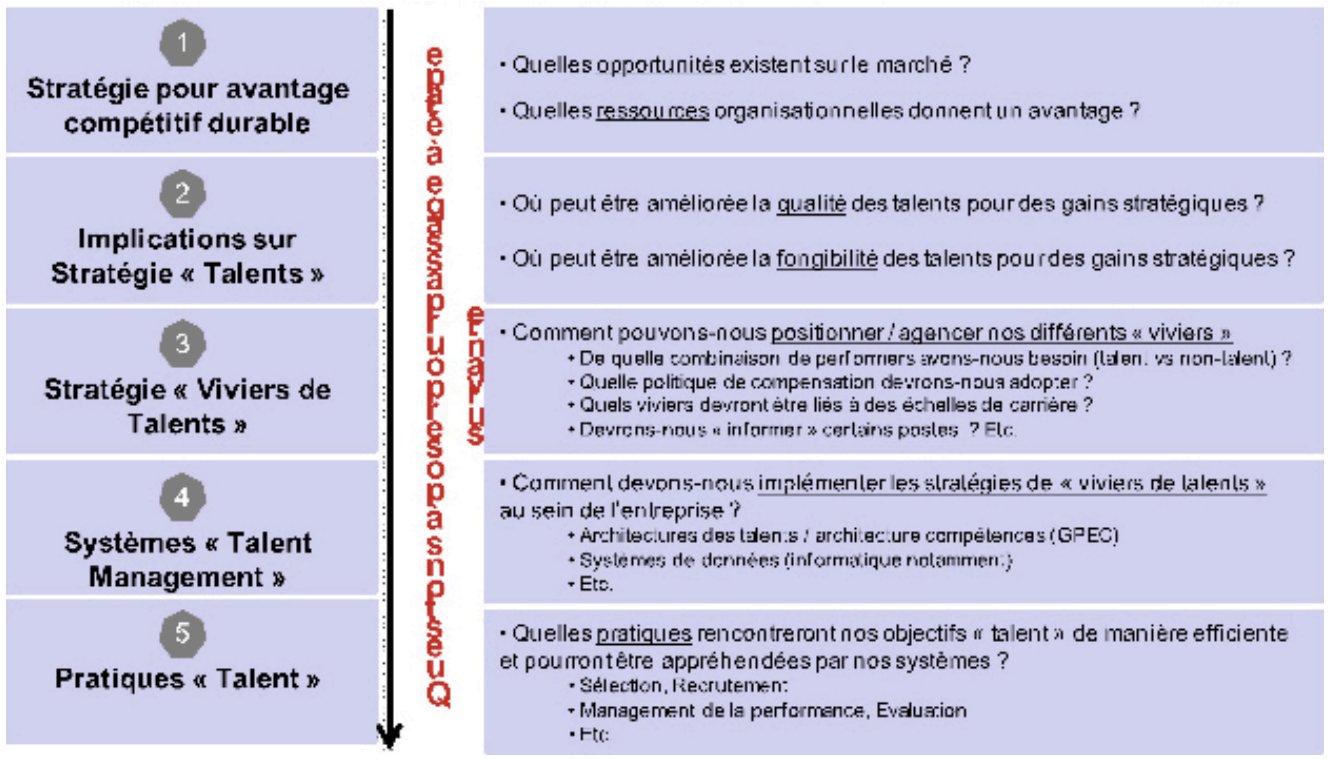

Les décisions en termes d'identification des viviers de talents ou encore d'allocation des ressources organisationnelles à l'un ou l'autre de ces viviers sont donc cruciales. Le management stratégique du talent ou talentship (Collings et Mellahi 2009; Lewis et Heckman 2006) va ainsi consister à construire une architecture du TM (figure 1) à plusieurs étages (Lewis et Heckman 2006) résultant d'une série de décisions issues des réponses apportées aux éléments suivants :

- Il s'agit d'abord de s'interroger sur (1) la stratégie offrant un avantage compétitif durable à l'organisation, à savoir celle à mettre en œuvre en réponse aux conditions externes du marché. Cela exige de savoir où se trouve l'entreprise dans le présent et où l'on souhaite qu'elle soit dans le futur pour définir ce que les auteurs nomment les «A positions» (Huselid et al. 2005). Celles-ci sont définies à partir de deux critères : l'impact stratégique de l'emploi/poste et son avantage compétitif au regard de sa création de valeur par rapport au business modèle ${ }^{1}$. Cette étape conduit alors à une segmentation de ces éléments par « viviers » de « talents pivots ».

- Les questions portent ensuite sur (2) l'alimentation des « viviers de talents », c'est-à-dire sur la manière de déterminer et répartir les ressources conformément aux besoins de l'organisation (McDonnell 2011). Néanmoins, il s'agit à cette étape de différencier les viviers entre eux, tous n'ayant pas le même degré de centralité stratégique, c'est-à-dire que tous les viviers ne sont pas des « viviers pivots ». Il sera utile de s'interroger sur la distribution ou les combinaisons nécessaires en termes de « $\mathrm{A}, \mathrm{B}$ ou $\mathrm{C}$ performers » (destinés à occuper respectivement les $\mathrm{A}$, $\mathrm{B}$ ou $\mathrm{C}$ positions) et sur les pratiques plus ou moins différenciées qui leur sont associées (en termes de compensations, de gestion de carrière, etc.).

- Les questions portent alors dans un troisième temps sur (3) les systèmes (gestion des compétences, SIRH, etc.) et pratiques (sélection, recrutement, formation, développement, etc.) de TM. Il s'agit d'évaluer l'efficience des systèmes et pratiques de l'organisation au regard des objectifs attendus. Cappelli (2008) propose par exemple un modèle du TM en « juste à temps » faisant le pendant au management de la chaîne logistique sur le mode d'un système d'approvisionnement continu (sur le marché interne et externe). 


\subsection{L'exploration du processus de création / élaboration d'une stratégie du TM ?}

Le talentship représente donc un cadre décisionnel qui a l'énorme mérite à la fois d'aider à la prise de décision en matière de TM et de structurer la démarche d'implémentation de ses différents dispositifs pratiques. Il centre son attention sur le processus de mise en æuvre du TM. Se faisant, les guides qu'il fournit tendent cependant à chosifier le processus du TM en donnant l'impression d'un «produit fini », objectivé, tenant son efficacité de la bonne mise en pratique des principes que le talentship recommande. Or, la consistance apparente de cet objet doit être relativisée à la lumière du processus de création / construction (allant de l'idée du projet jusqu'aux portes de son implémentation) qui engage un individu ou un groupe d'individus et l'ensemble de leur subjectivité.

L'aboutissement de ce processus, à savoir la décision d'implémentation de la stratégie de TM, résulte d'une confrontation de perceptions, représentations, imaginations différentes, de biais de sélectivité de l'information, de visions et images diversifiées (selon nation, organisation, culture, secteur d'activité, etc.) de la part des acteurs à l'égard d'une notion, le talent, qui est en elle-même source de compréhensions hétérogènes. Cette hétérogénéité est d'ailleurs soulignée par la littérature sur le TM lorsqu'elle indique que les prises de décisions se font très souvent sans cadre réellement bien structuré de la part des acteurs, sans totale considération des données clés et pertinentes, et biaisées la plupart du temps par les préférences, instincts, choix cognitifs ou encore biais personnels des parties-prenantes impliquées dans la prise de décision sur le TM (Vaiman et al. 2012).

Il existe donc, selon nous, un paradoxe dans la littérature entre d'un côté une inconsistance théorique du talent ouvrant aux divergences d'interprétation entre les acteurs, et de l'autre, une recherche d'objectivation de la démarche talent à partir d'un modèle d'aide à la décision et à la structuration pratique «normalisant ». Cet article s'inscrit dans cet entre-deux pour interroger les antécédents d'une stratégie de TM et comprendre la manière dont elle est élaborée par les acteurs dans un contexte organisationnel particulier. Cette perspective de recherche n'a, à notre connaissance, fait l'objet d'aucune attention dans la littérature sur le TM. Elle peut pourtant permettre de vérifier l'exclusivité supposée de l'alignement du TM à la stratégie de l'organisation, et d'identifier l'existence d'autres facteurs de contingence au cours de son processus d'élaboration.

L'entrée dans ce processus de création / construction d'une stratégie de TM peut alors permettre d'évaluer les capacités réelles de toute organisation à s'engager dans une stratégie de TM. Si les capacités organisationnelles de stratégie, de structure, de culture, d'exécution, de leadership ou encore d'innovation semblent affecter a posteriori l'efficacité du système de TM (Joyce et Slocum 2012), il est possible que ces éléments interviennent a priori, dès la mise en œuvre du processus de création / d'élaboration d'un dispositif de TM. Cette perspective peut ainsi nous permettre de vérifier la capacité supposée de toute organisation à s'engager dans une stratégie du TM et de réfléchir aux pré-requis éventuels qui en assureront la viabilité dans un contexte donné.

21 Investiguer dans ces directions implique donc de pouvoir être immergé avec des acteurs engagés dans ce processus de création / construction d'une stratégie de TM. Nous avons eu l'opportunité de réaliser une recherche-action d'une année avec le Directoire d'une ETI française de 3000 salariés. Cette approche exploratoire nous a permis de participer directement à ce processus et d'observer les facteurs de contingence qui influencent les décisions prises par les acteurs lors de son élaboration et qui déterminent in fine la viabilité de ce type de projet. Cette recherche-action dont les modalités vont être à présent discutées vise à répondre à la question de recherche suivante : quels sont les facteurs organisationnels qui interviennent au cours du processus d'élaboration d'une stratégie de TM ? Et en particulier, quels sont les facteurs qui sont susceptibles de mettre en échec l'élaboration d'une stratégie de TM ?

\section{Méthodologie}

Une recherche-action permet au chercheur, en se fondant sur une relation collaborative avec son « client » en vue de résoudre un problème pratique (Coghlan 2011 ; Rapoport 1970), d'être 
immergé dans le phénomène étudié. Si elle paraît adaptée aux exigences posées par notre problématique de recherche, cette approche mérite cependant d'être rigoureusement explicitée pour en légitimer sa scientificité (Brydon-Miller et al. 2003 ; Coghlan 2011 ; Eden et Huxham 1996 ; Stringer 2007). Nous présentons en conséquence ici le contexte de la recherche-action et les modalités de son déroulement.

\subsection{Le groupe Régional}

Le contexte dont est issue notre interprétation des résultats (Eden et Huxham 1996) est celui d'une Entreprise de Taille Intermédiaire (ETI) de 3000 salariés localisée en Vendée et désignée dans la suite de cet article par le «Groupe Régional » ${ }^{2}$. Il s'agit d'un groupe familial créé au tout début du 20ème siècle. La gouvernance, assurée jusqu'à la fin des années 90 par la famille fondatrice, a évolué vers un modèle composé de deux instances : un Conseil de Surveillance réunissant plusieurs générations de la famille fondatrice (actionnaires principaux) et un Directoire dirigé par un président extérieur à la famille ayant recruté dès son arrivée l'ensemble des huit cadres dirigeants qui le composent.

Il est utile de souligner la singularité de l'identité vendéenne à laquelle est attaché le groupe. Celle-ci se caractérise par une grande proximité entre patrons et ouvriers liée à un mécanisme socioculturel hérité des modes de relations entre châtelains et paysans (Champain et al. 1985 ; Raveleau 1998). Ces cinquante dernières années, le système industriel vendéen des petites et moyennes entreprises s'est structuré, à la manière de l'activité agricole d'autrefois, autour de quelques «propriétaires familiaux » (Raveleau 1998) tels que Fleury Michon, Sodebo, Gautier Meubles ou encore le groupe Bénéteau. La forte imprégnation de cette culture vendéenne inscrit les rapports sociaux et salariaux dans la continuité des relations paysans / propriétaires, à savoir sous le mode d'un patronage emprunt de respect, marqué par une forte culture religieuse (Champain et al. 1985) et soucieux de sa responsabilité à l'égard des salariés. Le Groupe Régional est imprégné de cette culture qui rejaillit sur ses valeurs de proximité, simplicité, humanité et performance.

Régional est structurellement organisé autour du siège qui regroupe les fonctions supports (135 collaborateurs) et de trois activités composées d'unités (agences) ou d'entités (usines ou centrales à béton) autonomes :

- Le négoce de produits matériaux (1 230 collaborateurs, façade ouest de la France) qui représente l'activité historique de l'entreprise et la part la plus importante du chiffre d'affaires en 2011. Ce métier de commerce en magasins ou en agences est marqué par une forte culture de groupe où prévaut la tradition de l'oral. L'activité rencontre au moment de la recherche une problématique de coûts (problème de rentabilité en termes de marge brute liée à la vente et aux achats de matériaux) et d'organisation de sa force commerciale. Il existe également une activité indépendante de négoce dans les Dom-Tom (215 collaborateurs) soumise à de fortes spécificités locales.

- Le béton (180 collaborateurs) qui est à forte intensité capitalistique et dont les synergies potentielles de marché avec le négoce sont peu exploitées. La difficulté de cette activité porte sur un maillage territorial des centrales à béton (ouest de la France) qui ne permet pas de couvrir l'implantation de la clientèle et qui, par conséquent, génère des coûts de transport importants.

- La menuiserie (940 collaborateurs), activité la plus récente du Groupe, dont le siège est en Bretagne et qui entretient avec le siège une relation de «holding ». Une dizaine d'usines de fabrication d'ouvertures composent cette activité prédominée par une forte culture industrielle marquée par la formalisation et l'industrialisation des processus. La problématique de cette activité repose sur la qualité des services proposés et les délais de livraison. De récentes acquisitions de deux usines en Amérique du Nord (300 collaborateurs) ont récemment été réalisées.

Le contexte de Régional a fortement évolué depuis une dizaine d'années. Le groupe a connu une forte croissance pour l'ensemble de ses activités (15\% par an depuis 2 ans) qui, de manière endogène et exogène, a conduit à un triplement des effectifs en l'espace de vingt ans (800 
personnes en 1992 pour près de 3000 personnes en 2011). A cet effet taille, le groupe a vu son modèle de gouvernance évolué lors de son introduction en bourse dans les années 90 , en passant d'un modèle familial à un modèle actionnarial (à fortes contraintes de gestion), et d'un management par les fondateurs à un management par une équipe de direction dont la problématique de succession se pose à moyen/long terme (5 à 10 ans). De plus, dans le mouvement de son implantation dans les départements d'outre-mer (8\% des effectifs en 2011), le groupe entame une internationalisation avec de nouvelles acquisitions en Chine (2008) et au Canada (2011) pour l'activité menuiserie (qui représente 300 collaborateurs).

Le groupe Régional s'interroge donc en 2011 sur le fait de disposer en interne des «talents » nécessaires à l'organisation pour relever les défis liés à l'évolution de la taille du groupe et de sa culture, et plus précisément pour assurer les nombreuses successions à venir (à différents niveaux managériaux). Les risques perçus en cas d'absence de gestion des talents en interne tiennent à la « dilution » des valeurs originelles de la culture PME du groupe que l'équipe dirigeante entend continuer à faire vivre, une « distanciation managériale » (malgré un renforcement de l'encadrement atteignant environ 250 managers) sous l'effet de la taille et de l'internationalisation du groupe qui est contraire aux valeurs de proximité et de simplicité auxquelles la famille est attachée ou encore, une « discontinuité » stratégique à moyen/long terme en l'absence de « talents maison » capables de succéder aux dirigeants actuels.

\subsection{Le cadre de la recherche-action}

L'équipe de direction a donc amorcé un travail sur le «talent » en recensant les postes clés et les hommes clés du groupe avec une identification de 230 hommes clés sur environ 350 managers. Cette démarche, stoppée face aux difficultés rencontrées dans l'exploitation des données, a conduit le Président du Directoire et le DRH à nous solliciter début 2011. La demande consistait à « accompagner la direction du Groupe Régional dans sa réflexion sur la 'gestion des talents Régional' (Recherche) et la mise en ceuvre de solutions adaptées (Action)». Nous avons ainsi adopté une démarche de recherche-action nous permettant à la fois de satisfaire la demande de notre « client » et de contribuer au développement de nouvelles connaissances théoriques en matière d'élaboration d'une stratégie de TM (Coghlan 2011 ; Eden et Huxham 1996; Rapoport 1970). Nous avons formalisé un partenariat d'une année (juin 2011 à juin 2012), intitulé « Recherche-action Régional / Audencia », précisant l'approche méthodologique retenue, les engagements et attentes respectifs (livrables par exemple), ou encore les modalités financières et logistiques du projet.

Le protocole de recherche a été construit sur la base d'une alternance de cycles itératifs et continus (1) de réflexion sur le talent et le TM, et (2) d'action en termes d'implémentation de solutions pratiques et spécifiques à l'organisation (Greenwood 2007 ; Lewin 1946 ; Susman et Evered 1978). La participation de chacun des acteurs associés au processus de rechercheaction était précisée (Lewin 1946 ; Reason et Bradbury 2001 ; Roy et Prévost 2013) et conduisait à distinguer les « acteurs décisionnaires » (membres du Directoire) et les « acteurs contributeurs » (managers opérationnels). Les huit acteurs décisionnaires, parce qu'engagés avec nous dans un processus dialogique d'élaboration de la stratégie de TM, représentent ici les véritables « co-chercheurs » du dispositif de recherche-action (Coghlan 2011). Notons que parmi eux, le DRH a également joué un rôle d'interface permanent entre le Directoire et nousmêmes pour nous permettre d'assurer au mieux le pilotage du dispositif au regard des enjeux politiques et individuels que ce dernier soulevait inévitablement.

Nous avons donc pleinement assumé dans ce dispositif une double posture à la fois de chercheur et de consultant (Eden et Huxham 1996; Susman et Evered 1978) : d'une part nous étions garant du regard réflexif porté sur le processus de création de la stratégie de TM, et ce, au fur et à mesure de son développement, et, d'autre part, nous participions activement au processus de décision lui-même (Roy et Prévost 2013). Notre expérience professionnelle en tant que consultant RH et chercheur nous a permis de «naviguer », tout au long du dispositif, entre cycles de réflexion et d'action. Nous avons également tenu un journal de recherche pour rendre rigoureusement compte, à chaque instant, de la nature de notre implication dans le dispositif et celle des « co-chercheurs » (au niveau théorique et pratique). 
Avant de travailler avec les membres du Directoire, nous avons consulté et analysé la documentation adressée par l'entreprise (plan stratégique des 3 dernières années, bilan social, enquête sur le climat social, organigramme, fiches de fonction, etc.). Ce travail nous a permis d'approfondir notre compréhension du contexte avant d'échanger avec chacun des huit membres du Directoire en juin 2011 au travers d'entretiens individuels semi-directifs d'une à deux heures. Notre posture n'était pas celle d'un « sachant » en TM, mais d'un accompagnateur neutre, à l'écoute et intéressé par leur vision à l'égard de l'élaboration d'une stratégie de TM (Stringer 2007). Ces entretiens, dont nous garantissions la confidentialité et qui ont été intégralement retranscris, nous ont permis (1) d'évaluer le degré d'information et d'adhésion de chacun à la démarche, (2) d'appréhender leur vision respective sur la problématique du talent à partir d'exemples concrets de salariés considérés comme talentueux (en précisant leur nombre, leurs caractéristiques ou encore leurs différences avec les « très compétents »), et, (3) de discuter les modalités du dispositif à engager.

Le recueil de ces données a donné lieu en juillet 2011 à une analyse, à partir d'une grille comparative, de leur vision du «talent » et du dispositif de gestion interne envisagé, mais également à une identification des divergences et résistances au sein du Directoire. Nous avons alors réalisé en août 2011 une restitution de ces éléments auprès des membres du directoire au cours d'une réunion de 3 heures. Cette dernière a permis (1) de partager notre compréhension de l'organisation, de son contexte et de ses enjeux, (2) de présenter les différentes approches théoriques sur le talent et leur(s) vision(s) respectives sur cette notion, pour, enfin, (3) définir et valider ensemble les modalités de la démarche retenue (figure 2) en termes d'étapes, d'actions, de réflexions ou encore de délais.

Figure 2. La démarche de recherche-action

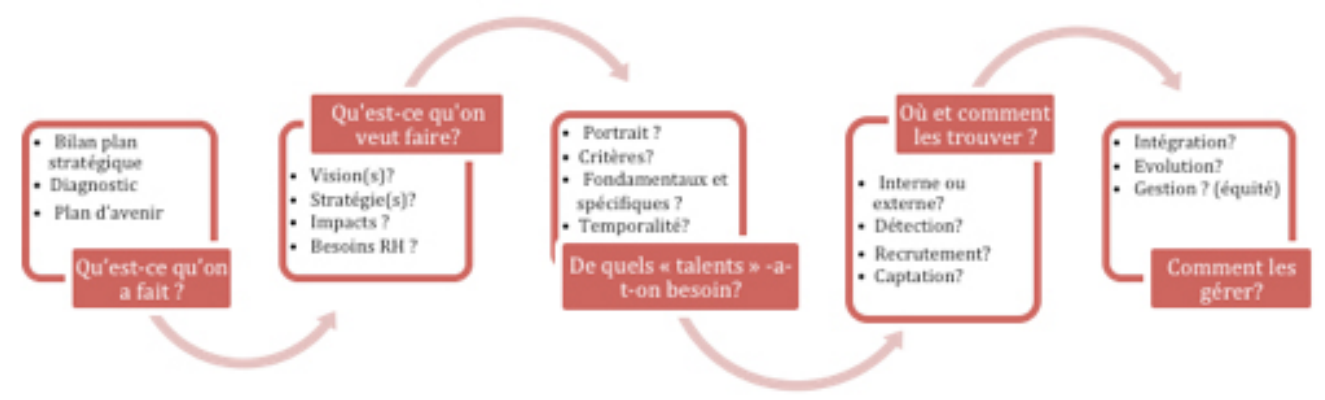

L'ensemble du groupe a validé ces différentes modalités de la recherche-action. Chacun des membres du Directoire s'est alors engagé à réfléchir, clarifier et à rédiger une proposition de plan stratégique en vue de nourrir l'élaboration de la vision stratégique du groupe (lors du séminaire de direction prévu fin août 2011) sur laquelle serait évalué l'alignement du TM lors de la prochaine réunion fixée en septembre/octobre 2011.

\subsection{L'évolution de la recherche-action}

La dégradation conjoncturelle des indicateurs économiques de l'entreprise, associée à la remise en question des savoirs et convictions induite par le projet, ont transformé la situation organisationnelle. Ces éléments ont généré des phénomènes particulièrement intéressants à prendre en compte dans une réflexion émergeante sur le processus de création d'une stratégie de TM (Eden et Huxham 1996). L'immersion qui a été la nôtre nous a non seulement permis d'observer les facteurs antagonistes à ce type de processus, mais également, comme nous y invite la littérature (Al Ariss et al. 2014), de restituer la dynamique subjective qui l'accompagne grâce au recueil qualitatif et longitudinal des expériences individuelles offert par la recherche-action.

A ce stade, face au recentrage des acteurs sur le court-terme et leur incapacité de projection stratégique à moyen/long terme, le protocole de recherche a évolué. Nous avons convenu avec le Président du Directoire et le DRH de les accompagner à cette clarification de la vision stratégique en faisant appel à un collègue chercheur en stratégie. L'objectif était à la fois d'apporter un regard neuf sur cette dimension stratégique, mais aussi de préserver la confiance instaurée avec le Président du Directoire pour la suite de la démarche en évitant de l'altérer 
par la confrontation directe et inévitable à laquelle conduirait cet exercice. Nous nous sommes donc placés à titre personnel, sur ce temps de clarification stratégique, dans une position d'accompagnateur et d'observateur.

Après divers échanges avec le DRH et notre collègue en vue d'apporter à ce dernier les clefs de compréhension du projet et de son contexte (jeux politiques notamment), a été conduite en septembre 2011 une série d'entretiens individuels d'une heure, intégralement retranscris, avec chacun des membres du directoire. Ces entretiens ne se centraient cette fois-ci que sur leur vision stratégique à moyen / long-terme pour le groupe et leur domaine d'activité. L'analyse de ces données a été complétée par celle des documents remis à notre demande par le Président du Directoire et le DRH (précédents bilans des plans stratégiques groupe et par activité). Une réunion préparatoire a été réalisée avec le Président du Directoire, le DRH et le DAF pour valider le déroulé et les modalités de la réunion de restitution auprès du directoire en octobre 2011.

Cette réunion, dont l'inscription dans le projet TM était rappelée en introduction, s'est déroulée sur une journée et en deux temps : « réflexion » le matin à partir d'une mise en commun des problématiques stratégiques, d'une présentation d'un rapport d'étonnement et d'apports méthodologiques ; pour, l'après-midi, basculer dans « l'action » d'une mise en perspective des orientations stratégiques retenues, d'une traduction chiffrée de celles-ci et d'une définition du plan d'actions à conduire dans le cadre du TM. Comme nous le discuterons plus loin, la dégradation continue de la conjoncture économique et l'exacerbation des tensions et résistances au sein du directoire, ont scellé l'incapacité des acteurs à partager une vision stratégique commune pour le futur (Coghlan 2011).

Nous avons donc convenu avec le DRH de laisser le temps de la réflexion stratégique aux acteurs pour retravailler sur ce point lors des réunions du directoire qui suivaient fin décembre 2011. Les difficultés étant les mêmes début 2012, et renforcées notamment par une réunion du Président du Directoire avec le conseil de surveillance début janvier 2012, nous avons décidé avec le DRH d'ouvrir la démarche aux managers intermédiaires (population cible du projet TM) pour à la fois laisser le temps au Directoire d'asseoir sa position stratégique et lui permettre de bénéficier d'une remontée d'informations. Cette étape a été conduite de février à mai 2012 par une approche multi-méthodologique (4 focus-groupes d'une durée de 3 heures chacun et 35 entretiens individuels semi-directifs d'une à deux heures) permettant la triangulation des données en vue de leur interprétation (Eden et Huxham 1996).

Encadré 1. Caractéristiques de l'échantillon des managers intermédiaires

Il est à noter que plus de $60 \%$ des collaborateurs repérés comme « talent » par le Directoire ont participé aux focus groupe et/ou aux entretiens individuels. Nous avons également sélectionné les salariés à interviewer de manière à garantir la représentativité de notre échantillon par rapport à la population totale. Celui-ci, représentant $10 \%$ de la population managériale (cible de l'étude), était composé de managers issus pour 6,1\% de l'activité Béton (contre 7,5\% en global), 9,1 \% des DOM (contre 8,4 \%), 33,3\% de la menuiserie (contre 30,7 \%), 30,3\% du négoce (contre $30,4 \%$ ) et $21,2 \%$ des fonctions supports au siège (contre $23,1 \%$ ). L'âge moyen de notre échantillon était de 45/46 ans, comme pour la population globale, avec néanmoins une sur-représentativité des interviewés de 40 à 45 ans et une sous-représentativité des interviewés de 35 à 40 ans. L'ancienneté moyenne de 12/13 ans était comparable à celle de la population globale, avec cependant une sur-représentativité dans l'échantillon des interviewés d'ancienneté de 7 à 10 ans et une sous-représentativité des salariés d'ancienneté de 25 à 30 ans. Enfin, notre échantillon était représentatif de la population managériale composée à $83 \%$ d'hommes et à $17 \%$ de femmes.

Nous avons alors réalisé une analyse du matériau discursif recueilli lors des focus groupes et entretiens individuels que nous avions enregistrés et intégralement retranscrits. Nous avons 
commencé par synthétiser les propos tenus par les participants à partir d'une matrice organisée autour des principales thématiques des guides semi-directifs que nous avions à la fois préparés pour les focus groupes et les entretiens individuels (vision entreprise, métier, enjeux et attentes personnelles). Un second niveau d'analyse nous a alors permis d'identifier les principales observations des managers intermédiaires à l'égard de l'entreprise (la vision globale, la culture, l'identité, l'organisation et le fonctionnement, le marché, la stratégie, le Directoire, le management, les collaborateurs, la vision du groupe demain). Ces éléments, dans leur recoupement avec l' analyse du travail effectué avec les membres du directoire, nous ont permis en troisième lieu de déceler les facteurs antagonistes à l'implémentation d'une stratégie de TM. L'ensemble de ces données a donné lieu à une présentation finale auprès du directoire fin juin 2012 lors d'une réunion de restitution de 3 heures. Cette réunion avait pour objectif (1) de souligner l'importance du TM au regard des résultats et témoignages issus de travaux présentés lors des conférences internationales sur le TM (académiques et professionnelles) auxquelles nous avions participé ; (2) d'apporter des clefs de compréhension sur les facteurs de succès ou d'échecs d'une stratégie de TM ; (3) de rendre compte des résultats de l'étude réalisée auprès des managers intermédiaires ; pour enfin (4) proposer des pistes d'action concrètes visant à leur permettre de dépasser les difficultés identifiées lors de la recherche-action en vue d'implémenter leur stratégie de TM.

\title{
3. Résultats
}

Cette recherche-action nous permet de rendre compte des facteurs de contingence au processus d'élaboration de la stratégie de TM du groupe Régional. Nous mentionnons ici les facteurs qui ont fait obstacle à l'aboutissement de ce processus, et donc à l'implémentation d'un dispositif de TM, pour nous amener à proposer, lors de la discussion de ces résultats, une série de réflexions préalables et complémentaires au modèle du talentship.

\begin{abstract}
"Demain, on sait qu'il y aura de nouveaux modes de consommation, de nouveaux modèles de construction avec de nouveaux canaux de distribution, mais on fait comme si ça n'existait pas et on n'anticipe pas ces évolutions... » (un membre du directoire)
\end{abstract}

Nous avons très rapidement constaté une incapacité des acteurs à énoncer la stratégie du groupe pour les années à venir. Celle-ci s'explique par un manque d'anticipation des profondes évolutions environnementales que le marché de la construction a connu. Ces évolutions portent par exemple sur l'apparition de nouvelles normes de construction, plus respectueuses de l'environnement (ex. : BBC, RT 2012, etc.), qui affectent la production et la commercialisation des produits de construction. Elles tiennent également à une concentration des acteurs de la construction qui conduit à la fois à réviser l'approche commerciale vis-à-vis de nouveaux décideurs et prescripteurs dans l'attribution des marchés de la construction (notamment les architectes), et à « brouiller » la lisibilité du Directoire sur la nouvelle logique concurrentielle.

"La chose qui me choque le plus est qu'on est effrayé par internet. On considère toujours notre approche client de la même manière, comme il y a 3 ou 4 ans... Mais le monde a changé et maintenant on doit intégrer les nouvelles technologies dans notre approche commerciale, mais quand on parle d'internet ou de réseaux sociaux... waouh, ce sont des gros mots! » (un manager intermédiaire)

Ces évolutions viennent impacter directement les métiers, compétences et talents dont le groupe aura besoin demain. Elles s'ajoutent à l'apparition d'un nouveau profil de « consommateur » qui, s'il avait hier un profil «d'artisan-bricoleur » fidèle à son enseigne et son magasin, est aujourd'hui un «technicien-gestionnaire » plus expert dans la connaissance des produits, plus exigeant en termes de services et de conseils proposés et plus volatile vis-à-vis de ses fournisseurs (comme le groupe Régional). Les nouvelles technologies (ex : smartphones, réseaux sociaux, etc.) n'ont fait qu'accompagné ce mouvement en dotant ce nouveau consommateur d'outils lui permettant de comparer et de trouver le meilleur produit au meilleur prix. Ce phénomène technologique vient bouleverser le mode de commerce traditionnel et accompagner la complexité de l'environnement concurrentiel du groupe Régional. 
"On ne vend plus simplement une plaque de placo, on vend une maison, c'est-à-dire un système de construction qui combine différents produits ensembles en vue d'atteindre une performance énergétique déterminée » (un membre du directoire)

Aussi, les acteurs sont mis en devoir d'énoncer une stratégie dans un environnement en pleine mutation dont ils n'ont pas anticipé les modalités : d'une part l'importance de l'innovation stratégique pour se différencier face à la saturation de l'offre, à l'exigence et l'infidélité des consommateurs, ou encore la primauté du marketing, et, d'autre part, l'hyper-concurrence du marché de la construction face à une reconfiguration du paysage concurrentiel et à de nouvelles solutions de construction plus «globales ». Les compétences techniques et comportementales qui sont nécessaires aux vendeurs dans ce contexte reposent, par exemple, sur sa connaissance globale du système de construction dans lequel un produit s'inscrit (et non plus seulement le produit), de ses performances, ou encore sur sa capacité à apporter un « conseil » à son client (pour l'attirer et le fidéliser).

«Tout le monde sait que nos métiers vont changer et que c'est inévitable, et que les personnes qui vont arriver dans l'entreprise dans 5 ou 10 ans auront une appréhension des nouvelles technologies que nos patrons [membres du directoire] n'ont pas » (un manager intermédiaire)

Le Directoire se trouve par conséquent en difficulté pour déterminer la stratégie susceptible d'offrir un avantage compétitif durable à l'organisation en réponse à ces nouvelles conditions du marché. Malgré le travail effectué avec notre collègue sur cette dimension stratégique (visant à poser les bases d'un diagnostic commun et d'une vision partagée de la situation ou encore de définir les critères de sélection des plans stratégiques du groupe), la démarche d'élaboration de la stratégie du TM reste bloquée à la première étape du modèle du talentship (cf. figure 1). N'étant pas en situation de définir les besoins futurs de l'organisation, le Directoire ne peut dès lors avancer dans une réflexion tenant à la définition des «A positions » (Huselid et al. 2005) sur laquelle repose la segmentation par « viviers » de « talents pivots».

"J'ai l'impression que chaque service est recroquevillé sur lui-même et qu'il vit de manière autonome (...) toutes les activités vivent dans leur propre monde » (un manager intermédiaire)

Il s'est avéré au cours de la recherche-action que cette panne de « vision stratégique » trouvait aussi ses origines dans l'histoire récente de l'entreprise : la crise économique de 2009. A ce moment, alors que la plupart de ses concurrents adoptaient une stratégie de restructuration de leurs activités, Régional a préféré faire le choix du maintien de l'emploi au nom des valeurs humanistes et de responsabilité sociale du groupe (renforcées par l'identité vendéenne). Cette décision stratégique s'avère représenter a posteriori, pour le Directoire, le premier «mauvais choix stratégique » de la direction depuis 15 ans. Celui-ci a conduit au maintien d'une configuration organisationnelle inappropriée aux besoins réels en ressources du groupe et a eu pour effet indirect d'altérer la dynamique collective en créant un sentiment diffus de sécurité dans l'emploi à la source de comportements de repli sur soi, de baisse d'implication et de limitation des prises d'initiatives. "L'atmosphère au sein du directoire n'est plus la même qu'avant, et je peux le dire car j'ai
beaucoup travaillé avec eux ces 11 ou 12 dernières années » (un manager intermédiaire)

Ainsi, en l'espace de deux ans, un manque de confiance stratégique s'est diffusé au sein des membres du Directoire et a été renforcé au moment de la recherche-action par les quatre crises intra-organisationnelles que le groupe rencontrait :

- Une crise de croissance « classique» sous l'effet de la taille et de la diversification du groupe qui amène les acteurs à s'interroger sur les choix d'investissements prioritaires, mais aussi sur le destin du fonctionnement « groupe » sous la forme soit d'une collection actuelle de «PME », soit d'un groupe ayant des synergies inter-activités.

- Une crise culturelle provoquée à la fois par le glissement d'une culture PME à une culture corporate, d'une culture commerciale portée par le négoce (culture de l'oral) à une culture industrielle portée par la menuiserie (culture de l'écrit), et enfin, d'une culture locale et vendéenne à une culture en voie d'internationalisation qui pose la question de la gestion 
multi-locale, des régions où développer son activité, ou encore de la création d'une direction internationale.

- Une crise de structure d'un groupe atomisé plutôt qu'unifié et marqué par un cloisonnement de ses différentes activités, antagoniste à la diffusion d'un « esprit talent ».

- Une crise managériale liée à l'hétérogénéité des pratiques selon les activités, les métiers, les entités et les managers, mais également liée à la problématique de succession de l'équipe de direction actuelle que nous avons observée et dont les collaborateurs perçoivent les dissensions internes.

Ce point est d'ailleurs un facteur essentiel pour expliquer les difficultés rencontrées par les acteurs dans l'élaboration d'une stratégie du TM. Nous avons constaté tout au long de la recherche-action une forme de désunion du Directoire, de conflits interpersonnels plus ou moins larvés qui ont conduit quelques mois après notre intervention à deux départs (le Président du Directoire et le Directeur Négoce). Les divergences portaient principalement sur la vision stratégique du groupe même si tout sujet en devenait un révélateur. Dès la première réunion de travail sur l'interprétation de la notion de «talent » nous avons pu constater la prégnance de ces divergences et les remises en question du leadership auxquelles elles conduisaient à chaque étape de la recherche-action. "On est complètement dans le brouillard et le problème c'est qu'on le sent [le Président du
directoire] totalement dans le flou par rapport à ce qu'on veut vraiment pour le futur. Il reste
toujours très vague parce qu'il a perdu sa vision pour l'avenir de l'entreprise » (un membre du
directoire)

Enfin, la démarche a également achoppé sur les divergences de visions à l'égard du talent au sein du Directoire. Si certains considéraient le talent comme dépendant du contexte professionnel dans lequel il est situé (en tant que personne), d'autres le liaient aux qualités intrinsèques de l'individu. Ainsi, malgré le travail de réflexion théorique conduit avec le Directoire, et parce que la démarche exigeait de clarifier préalablement la stratégie du groupe, la notion du talent pour le groupe est restée ténue pour les acteurs dans sa différenciation aux notions de « potentiel », « d'hyper-compétence » ou encore « d'Homme-clé », abstraite car définie plutôt en esprit (« une âme », « un truc de plus », etc.) que de manière concrète, variable selon le périmètre considéré (métier, fonction, territoire, etc.), et statique en l'absence de perspective dynamique.

L'ensemble de ces éléments représentent ainsi quelques uns des facteurs antagonistes à un processus d'élaboration d'une stratégie de TM. Ils illustrent l'incapacité organisationnelle de Régional à s'engager dans ce type de projet en raison d'une incapacité d'énonciation de la stratégie du groupe face à de mauvais choix passés, à un manque d'anticipation des évolutions du marché, à la traversée d'une période de crises (croissance, culture, structure, management et de leadership), ou encore d'une absence de définition préalable de ce que le « talent » représente pour l'organisation. Cela renvoie à une perception de manque de maturité organisationnelle pour engager une stratégie du TM qu'il s'agit à présent de discuter.

\section{Discussion}

Cette recherche-action nous permet de discuter quelques unes des voies méconnues par la littérature sur le TM au regard des quelques facteurs organisationnels qui apparaissent lors du processus d'élaboration de la stratégie du TM (Al Ariss et al. 2014). Elle permet également d'ouvrir une réflexion sur le talentship intégrant une série de questions préalables sur les capacités organisationnelles requises pour implémenter une stratégie de TM (Collings et Mellahi 2009 ; Lewis et Heckman 2006).

Les résultats confirment d'abord la contingence de l'interprétation du talent relevée par la littérature sur le TM (Gallardo-Gallardo et al. 2012 ; Nijs et al. 2014 ; Tansley 2011). Ils illustrent la différence d'interprétation du talent selon la position qui est celle de l'appréciateur dans l'organisation, le type d'activité dans laquelle celui-ci est situé ou celle où il se trouve amené à considérer le talent, ou encore le fait que le talent soit pour lui une potentialité 
avérée ou à développer (talent présent / futur talent). Nous rejoignons donc la conclusion de la littérature sur l'impossibilité de définir universellement cette notion, mais prenons de la distance à l'égard de travaux laissant supposer une définition partagée du talent entre les différentes parties-prenantes de la même organisation (Al Ariss et al. 2014). Non seulement les aspects d'ordre cognitif entrent en jeu dans l'interprétation du talent des individus impliqués dans la prise de décision sur le TM (Vaiman et al. 2012), mais également les aspects d'ordre subjectif, émotionnel et affectif liés à l'histoire, les convictions, ou encore les désirs et enjeux personnels qui leur sont propres.

Une piste de recherche fructueuse consisterait à prendre en considération la subjectivité individuelle à l'œuvre dans une stratégie de TM, c'est-à-dire à questionner les clefs de motivation intrinsèque et la manière dont elles en affectent le processus d'élaboration. L'appel au « talent » qui nous a été adressé par Régional semble notamment, au regard des fragilités organisationnelles qui se sont révélées tout au long de la démarche de recherche-action, masquer ces enjeux individuels d'ordre imaginaires et subjectifs chez les décisionnaires qu'il serait utile de questionner (le fantasme du «talent-sauveur », les mécanismes projectifs de soi dans le «talent », la mode du talent, etc.).

Nos résultats montrent également qu'un alignement exclusif du TM à la stratégie (Boudreau et Ramstad 2004 ; Collings et Mellahi 2009 ; Lewis et Heckman 2006 ; McDonnell 2011 ; Vaiman et al. 2012) n'est pas suffisante pour assurer le succès de son implémentation. Le cas Régional suggère que ce succès dépend aussi de l'alignement du TM sur (1) une structure organisationnelle qui, contrairement à une organisation en « silos », favorise l'épanouissement des talents grâce aux opportunités de mobilité (professionnelle, évolution de carrière, etc.) ; (2) une culture organisationnelle porteuse d'un état d'esprit talent (Cappelli 2008) qui dépasse les frontières fonctionnelle, sectorielle ou encore géographique ; ou encore (3) les communautés de pratiques managériales et de leadership cohérentes qui renforcent l'épanouissement des talents par la synergie et le travail collaboratif, la décentralisation du pouvoir, des responsabilités et des décisions à certaines positions de l'organisation. De futurs travaux méritent d'être conduits pour évaluer plus précisément les différentes dimensions sur lesquelles une stratégie de TM doit être alignée lors de son élaboration afin de garantir le succès de son implémentation.

Cette perspective conduit plus précisément à questionner les capacités organisationnelles (Joyce et Slocum 2012) propres à une organisation qui souhaite s'engager dans une stratégie de TM. Il ne s'agit pas seulement d'évaluer a posteriori l'influence des capacités organisationnelles sur la performance d'un dispositif de TM dans une organisation donnée, mais d'évaluer a priori la nature de ces capacités lors du processus d'élaboration d'une stratégie de TM pour en déterminer la viabilité. Le cas Régional est une illustration du fait d'une part que toute organisation ne paraît pas forcément armée pour s'engager dans ce type de démarche, d'autre part qu'il serait utile de réaliser un diagnostic préalable sur ses capacités à mener à son terme une stratégie de TM, et enfin qu'il serait souhaitable de pouvoir identifier les facteurs organisationnels susceptibles d'enrayer ou au contraire d'assurer l'implémentation de ce type de dispositif. Des travaux de recherche centrés sur les pré-requis organisationnels à une stratégie de TM permettraient alors d'avancer dans la compréhension à la fois théorique et pratique des conditions d'implémentation du TM.

Cette discussion nous conduit ainsi à proposer un nouvel étage de réflexions préalables au talentship (Boudreau et Ramstad 2004 ; Cappelli 2008 ; Cappelli et Keller 2014 ; Lewis et Heckman 2006) en vue de permettre aux décisionnaires du TM d'anticiper la capacité éventuelle de leur organisation à s'engager dans une stratégie de TM. Ces réflexions s'organisent autour d'une série de questions portant sur 5 niveaux d'analyse indépendants et complémentaires sur lesquels doit être évalué l'alignement de la stratégie du TM :

- La stratégie : au-delà de la nécessité de clarifier et d'énoncer la stratégie ou plans stratégiques de l'organisation, les acteurs devront s'interroger sur (1) l'alignement des leaders sur la stratégie organisationnelle, (2) la compréhension de cette vision stratégique par l'ensemble des parties-prenantes de l'organisation, et (3) la conformité du fonctionnement organisationnel à ces principes stratégiques. 
- La structure : il s'agit ici d'évaluer la structure organisationnelle pour (1) déterminer la capacité de l'organisation à offrir des opportunités de mobilités professionnelles et/ou de transférabilités des compétences, (2) libérer et assurer les synergies entre activités, services et fonctions en termes de pratiques et de processus de gestion des talents, (3) repérer et prévoir les pénuries en talent éventuelles en termes de compétences, de marchés et de délais auxquels l'organisation peut faire face au regard de ses choix stratégiques, et (4) savoir comment accompagner efficacement le changement au sein de l'organisation.

- La culture : les décisionnaires devront également (1) évaluer la capacité de promotion et de diffusion de l'état d'esprit talent (Cappelli 2008) au travers des frontières géographiques et fonctionnelles de l'organisation, et (2) s'assurer que leur comportement est consistant et cohérent avec les valeurs qu'ils promeuvent et souhaitent promouvoir.

- Le leadership : l'élaboration d'une stratégie de TM menée à bien repose sur la capacité de l'équipe de direction à (1) évaluer et s'assurer de la cohésion et de l'engagement de chacun de ses membres, (2) s'accorder sur une interprétation organisationnelle du « talent », et (3) porter et diffuser l'esprit talent dans ses activités.

- Le management : il s'agit ici d'évaluer (1) l'hétérogénéité éventuelle des pratiques existantes qui sont susceptibles d'altérer l'efficacité de la gestion des talents, (2) l'adhésion des managers à l'interprétation organisationnelle du talent, (3) la capacité et la maturité de ces managers à gérer les talents, et (4) la compréhension collective des dynamiques intergénérationnelles et interculturelles du talent.

Ces éléments, sans que nous prétendions ici en avoir dressé l'exhaustivité, constituent à la lumière du cas Régional quelques unes des interrogations préliminaires auxquelles les décisionnaires auront à répondre au cours du processus d'élaboration d'une stratégie de TM. Ils représentent les 5 dimensions du diagnostic préalable que les décisionnaires auront à conduire sur les capacités organisationnelles de mise en œuvre du TM. Cette proposition exploratoire d'évaluation des conditions organisationnelles requises au TM peut ainsi représenter un cadre d'analyse complémentaire au talentship pour aider à la prise de décision lors de l'élaboration d'une stratégie de TM et garantir le succès de son implémentation.

\section{Conclusion}

Cet article présente une réflexion émergente sur le processus de création / élaboration d'une stratégie de TM actuellement méconnue dans la littérature sur le TM. Il explore les facteurs organisationnels contingents à ce processus par l'intermédiaire d'une recherche-action d'une année conduite dans un contexte organisationnel non anglo-saxon d'une ETI française (Chuai et al. 2008 ; Collings et al. 2009 ; Mellahi et Collings 2010). Les résultats de cette recherche nous amènent à relativiser deux présupposés principaux de la littérature sur le management stratégique du talent, à savoir l'exclusivité supposée de l'alignement du TM à la stratégie de l'organisation et la capacité que toute organisation aurait à mettre en œuvre ce type de dispositif (Thunnissen et al. 2013).

Cette étude montre qu'au-delà de la nécessaire clarification stratégique évoquée par la littérature, divers facteurs interviennent dans l'élaboration d'une stratégie de TM : la nature des décisions stratégiques passées, les variables environnementales internes (structure, culture, leadership, management, etc.) et externes (société, marché, technologie, etc.), l'histoire de l'organisation et de ses acteurs, ou encore les désirs ou ambitions personnels. Le caractère exploratoire de cette démarche nécessite néanmoins de mener de futurs travaux pour à la fois approfondir la part de ces facteurs dans l'élaboration d'une stratégie de TM, et en particulier la manière dont les expériences individuelles subjectives traversent ce processus (Al Ariss et al. 2014), mais également pour vérifier la validité et la généralisation de ces résultats à d'autres contextes organisationnels (Al Ariss et al. 2014 ; Stahl et al. 2012).

Ces travaux de recherche permettraient d'enrichir le champ des connaissances académiques et professionnelles sur les conditions ou capacités organisationnelles requises (Joyce et Slocum 2012) pour élaborer avec succès une stratégie de TM. Ils permettraient d'approfondir la réflexion sur un nouvel « étage » de réflexions du talentship (Boudreau et Ramstad 2004 ; 
Cappelli 2008 ; Cappelli et Keller 2014 ; Lewis et Heckman 2006) de cet article en proposant un modèle de diagnostic préalable sur les capacités ou compétences organisationnelles d'implémentation du TM. Cet outil permettrait à n'en pas douter d'éviter à certaines organisations de succomber à une «mode managériale » pour laquelle elles ne sont par armées.

\section{Bibliographie}

Al Ariss, A., Cascio, W. F., et Paauwe, J. (2014), Talent management: Current theories and future research directions, Journal of World Business, vol. 49, $\mathrm{n}^{\circ}$ 2, p. 173-179.

Boudreau, J. W., et Ramstad, P. M. (2004), 'Talentship': a decision science for HR. Why HR measurement must focus on informing better talent decisions, Strategic HR Review, vol. 3, n ${ }^{\circ}$ 2, p. 28-31.

Boudreau, J. W., et Ramstad, P. M. (2007), Beyond HR: The New Science of Human Capital.

Brydon-Miller, M., Greenwood, D., et Maguire, P. (2003), Why action research?, Action research, vol. 1, $\mathrm{n}^{\circ} 1$, p. 9-28.

Cappelli, P. (2008), Talent Management for the Twenty-First Century, Harvard Business Review, vol. March, p. 74-81.

Cappelli, P., et Keller, J. (2014), Talent Management: conceptual approaches and practical challenges, Annu. Rev. Organ. Psychol. Organ. Behav., vol. 1, n 1, p. 305-331.

Champain, L., Clément, J.-Y., et Regourd, F. (1985), Le syndicalisme chrétien en Vendée.

Chuai, X., Preece, D., et Iles, P. (2008), Is Talent Management Just 'Old Wine in New Bottles'? The Case of Multinational Companies in Beijing, Management Research News, vol. 31, $\mathrm{n}^{\circ}$ 12, p. 901-911.

Coghlan, D. (2011), Action research: Exploring perspectives on a philosophy of practical knowing, The Academy of Management Annals, vol. 5, $\mathrm{n}^{\circ}$ 1, p. 53-87.

Collings, D. G., McDonnell, A., et Scullion, H. (2009), Global talent management: the law of the few, Poznan University of Economics Review, vol. 9, $\mathrm{n}^{\circ}$ 2, p. 5-18.

Collings, D. G., et Mellahi, K. (2009), Strategic Talent Management: a Review and Research Agenda, Human Resource Management Review, vol. 19, p. 304-313.

Collings, D. G., Scullion, H., et Vaiman, V. (2011), European perspectives on talent management, European Journal of International Management, vol. 5, $\mathrm{n}^{\circ}$ 5, p. 453-462.

Eden, C., et Huxham, C. (1996), Action research for management research, British Journal of Management, vol. 7, $\mathrm{n}^{\circ}$ 1, p. 75-86.

Gallardo-Gallardo, E., Dries, N., et Gonzalez-Cruz, T. (2012), What is Meant by 'Talent' in Business?, in Workshop on Talent Management, EIASM, Bruxelles.

Greenwood, D. J. (2007), Pragmatic action research, International Journal of Action Research, vol. 3, $\mathrm{n}^{\circ} 1+2$, p. 131-148.

Huselid, M. A., Beatty, R. W., et Becker, B. E. (2005), "A Players" or "A Positions"? The Strategic Logic of Workforce Management, Harvard Business Review, vol. December, p. 110-117.

Joyce, W. F., et Slocum, J. W. (2012), Top management talent, strategic capabilities, and firm performance, Organizational Dynamics, vol. 41, n 3, p. 183-193.

Lewin, K. (1946), Action research and minority problems, Journal of social issues, vol. 2, n 4, p. 34-46.

Lewis, R. E., et Heckman, R. J. (2006), Talent Management: a Critical Review, Human Resource Management Review, vol. 16, p. 139-154.

McDonnell, A. (2011), Still Fighting the "War for Talent"? Bridging the Science Versus Practice Gap, Journal of Business and Psychology, vol. 26, $\mathrm{n}^{\circ}$ 2, p. 169-173.

Mellahi, K., et Collings, D. G. (2010), The barriers to effective global talent management: the example of corporate elites in MMEs, Journal of World Business, vol. 45, n 2, p. 143-149.

Meyers, M. C., et van Woerkom, M. (2014), The influence of underlying philosophies on talent management: Theory, implications for practice, and research agenda, Journal of World Business, vol. 49, $\mathrm{n}^{\circ} 2$, p. $192-203$.

Meyers, M. C., van Woerkom, M., et Dries, N. (2013), Talent - Innate or acquired? Theoretical considerations and their implications for talent management, Human Resource Management Review, vol. $23, n^{\circ} 4$, p. 305-321.

Michaels, E., H.Handfield-Jones, et Axelrod, B. (2001), The War for Talent. 
Nijs, S., Gallardo-Gallardo, E., Dries, N., et Sels, L. (2014), A multidisciplinary review into the definition, operationalization, and measurement of talent, Journal of World Business, vol. 49, $n^{\circ} 2$, p. $180-191$

Rapoport, R. N. (1970), Three dilemmas in action research with special reference to the Tavistock experience, Human Relations, vol. 23, n 6, p. 499-513.

Raveleau, B. (1998), Les entrepreneurs industriels du bocage vendéen : essai sur la contribution des chefs d'entreprise au développement industriel du nord-est de la Vendée, CNAM.

Reason, P., et Bradbury, H. (2001), Handbook of action research: Participative inquiry and practice.

Roy, M., et Prévost, P. (2013), La recherche-action : origines, caractéristiques et implications de son utilisation dans les sciences de la gestion, Recherches qualitatives, vol. 32, $\mathrm{n}^{\circ}$ 2, p. 129-151.

Silzer, R., et Church, A. H. (2009), The Pearls and Perils of Identifying Potential, Industrial and Organizational Psychology, vol. 2, p. 377-412.

Silzer, R., et Church, A. H. (2009), The Potential for Potential, Industrial and Organizational Psychology, vol. 2, p. 446-452.

Stahl, G., Björkman, I., Farndale, E., Morris, S. S., Paauwe, J., Stiles, P., Trevor, J., et Wright, P. (2012), Six principles of effective global talent management, Sloan Management Review, vol. 53, $\mathrm{n}^{\circ}$ 2, p. 25-42.

Strack, R. (2011), Creating People Advantage 2011: Time to Act: HR Certainties in Uncertain Times.

Stringer, E. T. (2007), Action research.

Susman, G. I., et Evered, R. D. (1978), An assessment of the scientific merits of action research, Administrative science quarterly, p. 582-603.

Tansley, C. (2011), What Do We Mean by the Term "Talent" in Talent Management, Industrial and commercial training, vol. $43, \mathrm{n}^{\circ} 5$, p. 266-274.

Thévenet, M., et Dejoux, C. (2010), La gestion des talents : La GRH d'après-crise, Management Sup, Dunod ed.

Thunnissen, M., Boselie, P., et Fruytier, B. (2013), A review of talent management :'infancy or adolescence ?', The International Journal of Human Resource Management, vol. 24, n 9, p. 1744-1761.

Vaiman, V., et Collings, D. G. (2013), Talent management: advancing the field, The International Journal of Human Resource Management, vol. 24, n 9, p. 1737-1743.

Vaiman, V., Scullion, H., et Collings, D. (2012), Talent management decision making, Management Decision, vol. 50, n 5 , p. 925-941.

\section{Notes}

1 Les emplois en « position B » sont ceux qui ont un impact stratégique mais apportent peu d'avantage compétitif et les emplois en « position $\mathrm{C}$ » qui ont peu d'impact stratégique et d'apport compétitif

2 L'entreprise a souhaité conserver l'anonymat dans le cadre de toute diffusion publique au regard notamment des difficultés que la démarche a soulevé en interne et des conséquences qui ont suivi pour certains des acteurs de la démarche.

\section{Pour citer cet article}

Référence électronique

Mickael Naulleau, «Elaboration d'une stratégie de management du talent : quelques conditions de réussite illustrées par une recherche-action », Finance Contrôle Stratégie [En ligne], 18-3 | 2015, mis en ligne le 26 octobre 2015, consulté le 27 octobre 2015. URL : http://fcs.revues.org/1659

\section{À propos de l'auteur}

Mickael Naulleau

mnaulleau@audencia.com

\section{Droits d'auteur}




\section{Résumés}

La littérature sur le Management du Talent (TM) présuppose à la fois un alignement exclusif du TM sur la stratégie de l'organisation et une capacité de toute organisation à s'engager dans une stratégie de TM. Cet article interroge ces principes en explorant les facteurs organisationnels de contingence au processus de création / d'élaboration d'une stratégie de TM à partir d'une recherche-action menée pendant un an avec la direction d'une Entreprise de Taille Intermédiaire (3 000 salariés). Il propose un cadre d'analyse complémentaire au modèle décisionnel du talentship orienté sur un diagnostic préalable des capacités organisationnelles à l'implémentation d'une stratégie de TM.

The literature on Talent Management (TM) presupposes an exclusive alignment of TM with the organization's strategy, and the ability of any organization to engage a TM strategy. This article examines these principles by exploring the organizational contingency factors involved in the process of creation / development of TM strategy. We carried out a oneyear Action-Research with the business leaders of a French medium-sized company (3000 employees). We propose a complementary analytical framework to the decision model of Talentship which is focused on a prerequisite diagnosis on organizational capacities in TM strategy implementation.

\section{Entrées d'index}

Mots-clés : Management du talent, Stratégie, Talentship, Recherche-Action. Keywords : Talent Management, Strategy, Talentship, Action Research. Code JEL : L21 - Business Objectives of the Firm 